\title{
RFID Based Material Sorting System using Siemens DCS Controller
}

\author{
L.Chitra, Sunkrutha S, Prashant Pradhan
}

\begin{abstract}
The SIMATIC RF200 IO-link is an inductive identification system module which is compatible with the ISO 15693 standard and this is specifically intended to be used in the industry for the control and optimization of materialistic flow. Here the field bus distributed control system integrates with the RFID material this makes the sorting system more controlled and flexible. The system can be utilized for energy management also. The system can be extended by using it for real time power monitoring and control using dcs from multiple power sources. The profinet and profibus cables are used as bus interface here to interlace the simatic PCS 7 with the RFID and the reader.
\end{abstract}

\section{Keywords : DCS mechanism, SIMATIC,SCADA.}

\section{INTRODUCTION}

The growing need for reduced latency and immediate response time foe industrial application led to the development of DISTRIBUUTED CONTROL SYSTEM (DCS). DCS is widely used in industrial environment where multiple process needs to be executed simultaneously without any time lag or power issues. This kind of multi- tasking is achieved with the help of distributed control system. Material sorting plays an important role in the industrial atmosphere. It is primarily done with hand sorting, machine sorting and sensing methodologies. Here we intend to do an automated sorting methodology using machine-based methods. The SIMATIC manager software helps to linking the PCS 7 hardware module with the rfid. The pcs 7 is used in both production and process engineering in manufacturing and hybrid industries. The components used here include a conveyor belt, a photo electric proximity sensor, stepper motor, ET200 PA smart, profinet bus interface, pcs \& smart, RFID. These components are interlinked to stimulate a hybrid sensing of components moving in the conveyor belt there by enabling the operation of segregation and material sorting.

\section{COMPONENTS ENABLING MATERIAL SORTING}

\section{A. PCS7:}

The PCS 7 is a siemens controlled which acts as a central processing unit for the DCS. The quality assurance of

Revised Manuscript Received on December 30, 2019.

Dr.L.Chitra, *, Associate Professor and Head, Department of EEE, Aarupadai Veedu Institute of Technology, Chennai, India.

Sunkrutha S, UG Student, Department of Mechatronics, Aarupadai Veedu Instituite of Technology, Vinayaka Missions Research Foundation, Chennai, India.

Prashant Pradhan, *, UG Student, Department of Mechatronics, Aarupadai Veedu Instituite of Technology, Vinayaka Missions Research Foundation, Chennai, India.
SIEMENS PCS 7 is for the whole system. This appears in contrast to the Scada system which comes in a package of bundled individual components whose functional range is not co-ordinated. The SIMATIC PCS 7 processor is operated by means of graphical user interfaces with context dependent and user specific views. It has a greater flexibility compared to other systems due to the modularity of hardware and software components and hence it can be used for all process control systems. The capacity of this module ranges from a single small system to a multi user system consisting of multiple operator stations and servers associated with it in a plant-based networking. Moreover, the PCS 7 can be intertwined with components from other manufacturers as well other than SIEMENS. This is because the system architecture and communication are open. The data which are to be processed are accessible only on the basis of standardized interfaces and is available company wide. The communication to this DCS controller takes place via standardized professional ethernet and PROFIBUS. Terminals are user defined and are available in all the operator stations. Windows based PCS 7 servers enable remote monitoring and diagnostics. The maintenance of the system can be achieved through internet or intranet. The smart field devices (sensors and actuators) are controlled using SIMATIC Process Device Manager. Here PCS 7 is used as it is intrinsically safe the controller which offers high safety. This system also has a self-monitoring function that reacts to the faults of the system and the system failures.

\section{B. SIMATIC manager software:}

The simatic manager software represents the central software package which is used to program and instigate control solutions to the user by accessing the PCS 7. The simatic manager is linked to all the other application connected to the DCS and then the block is introduced in the CFC chart. The CFC editor and the SFC editor are inbuilt packages in the SIMATIC Manager software. The operator station and all the other system configuration are liked with the SIMATIC Manager software installed in the user computer which is then linked to the PCS 7 using he profinet bus interface. The software has features such as testing of automation system, diagnosing plant failure, simulation of program. Here the programming is featured using functional blocks which uses logic boxes familiar to Boolean algebra. Complex functions can be represented directly through aggregation with the functional blocks.

The functional blocks are available in the inbuilt library of the CFC's of the SIMATIC Manager. The functional block diagram of DCS is similar to the 


\section{RFID Based Material Sorting System using Siemens DCS Controller}

ladder logic diagram of PLC. Here all the operations are segregated into individual functional blocks and then the inputs and the outputs of that particular functional blocks are identified and then the functional logic is produced in the CFC chart.

\section{RFID components and their functions:}

Radio frequency identification is a wireless communication medium which is achieved through electrostatic or electromagnetic coupling of radio frequency waves. The RFID components consists of a scanning receiver, trans receiver and a transponder. It uses Radio frequency wave to detect the material and thus enabling material sorting in the conveyor line. There are three main types of RFID systems they are low frequency (LF), high frequency (HF) and ultra-high frequency (UHF). Microwave RFID is also present in today's industrial usage. Here we use RF200 high frequency RFID to meet the necessary condition. The RF 200 Series SNAP modules are designed to work with the ATMEL ATmega128RFA1 chip. RFID systems is one of the key technologies used in the industrial sector for the Digital Enterprise. In the area of RF identification systems, Siemens offers a wide range of collection that provides the perfect solution for all the application. The connection to the cloud-based, open IoT operating system enables Digital Factory and Industrialization-4.0. Thus, offering enhanced transparency, efficiency, and flexibility.

Application and overview of ISO transponders used in RF200:

A transponder is a dual acting mechanism that collects and transfer radio waves at a given density area. After receiving the signal, the transponder will be able to broadcast the signal at a different frequency range. The transponders are used in the industrial sector in Assembly and handling area for the identification of work piece carriers particularly in small assembly lines. The RFID system automatically supplies the data read by the transponder. The transponder can read can read unique identification numbers (UID) as well as and any user data of a predefined memory. The data are displayed in the process image of the controller as soon as a transponder is in the field of a reader or spots the work piece.

\section{CPU AS 410 Smart Controllers:}

Any The AS 410 system interfaced with the SIMATIC PCS 7 enables user to efficiently perform process control and can be used in all domains due to its versatility. For specific requirements the CPU AS410 can be configured in many types. The AS 410 system is designed powerful, versatile and rugged for the process industry. It is the fastest and the most powerful controller available in the market. It covers all application scopes and performance ranges. The controller's innovative dimensioning notion allows it to be of highly flexible and ready for use for standard, fault-tolerant and fool impervious applications in all performance categories. It has a 48 Mega Byte integrated load memory. It has an additional protection of Conformal Coating on electronics component making it robust free. No run or stop switch is provided making it prone to less risk for accidental switching of the controller. CPU reboots automatically in case of unforeseen stop or defect thereby, reducing blackout time in critical situation. It operates without backup battery.

\section{E. ET200SP - distributed I/O station:}

The ET 200SP is a Siemens developed product and it is one of the most popular remote I/O units used on the market today. A remote $\mathrm{I} / \mathrm{O}$ is a two-ended configuration where one end consists of a set of input and output modules which are installed close to the sensor and actuators on the factory floor and the other end is connected to the DCS in the control room by a single cable (PROFIBUS or PROFINET). With this network configuration, the sensors and actuators send the signals to the remote $\mathrm{I} / \mathrm{O}$ and then the remote $\mathrm{I} / \mathrm{O}$ takes those signals and sends them over to the DCS via a single cable.

This way, instead of running all of the cables directly to the DCS only one cable is used to connect the field devices to the control room. The SIMATIC ET 200SP offers maximum usability, compact design, and impressive performance. It offers free choice of the PROFINET connection via bus adapter and no tools are needed for wiring due to the inbuilt push-in technology and NC contacts with the associated conductor opening. It is noise-resistant due to short signal and encoder wiring. SIMATIC ET200SP with CPU allows intelligent pre-processing in the place of higher-level controller.

\section{F. Conveyor Belt:}

A conveyor belt is the transporting part of any belt conveyor system. A belt conveyor system consists of two or more pulleys and gear shafts, with an endless loop of carrying medium which acts as a transporter. The pulleys are powered in order to move the belt and the material on the belt forward The powered pulley is called the driver pulley while the unpowered pulley is called the idler or driven pulley. The conveyor belt acts as transporting medium where the material sorting takes place.

\section{G. Photo electric proximity sensor:}

Photo electric proximity sensor is a non-contact type of sensor. It thereby senses the presence of nearby object without any physical contact. A proximity sensor often emits an electromagnetic field radiation and identifies the changes in the return signal. Proximity sensors have high reliability and long functional life due of the absence of mechanical parts and nonexistence of physical contact between the sensor and the sensed object. Proximity sensors are used for a wide range of industrial and manufacturing applications where they are used to detect the presence of objects or materials and can either initiate some action or flag their presence or absence. Photoelectric proximity sensors operate on the basis of light irradiance and depending on the change of intensity of light. The two basic types of photoelectric sensors are reflective type and through-beam type. Reflective sensors exert by discharging a beam of light which when striking the object gets reflected back to the detector. In case of Through-beam sensors they have two separate units, an emitter and a separate receiver or detector. When an object breaks the light beam, the detector notes down this break modification during transmittance.

\section{H. Stepper Motor Driver:}

The process of converting a 
electrical power in to mechanical power is known as stepper motor driver. It enables error free rotation of the belt drives and the gears. It divides the full rotation of the armature into number of sub-dividing steps. In addition to that it is a brushless and synchronous electric motor. The stepper motor enables proper control in its rpm feedback is automatically generated to the motor circuit. Stepper motors are similar in construction to the switched reluctance motors. The stepper motor makes use of magnetic field theory to rotate the shaft when electricity is pulsated through it. The stepper motor usually completes one revolution in 24 steps making it move $15^{\circ}$ to complete one revolution. These are used in electrical gauges and motor tooling in automated production equipment and industrial atmosphere. Using stepper motor there exists high likeliness to achieve very low speed synchronous rotation with a load that is directly coupled to the shaft. A wide range of rotational speeds can also be achieved as the speed of the motor is directly proportional to the frequency of the input pulses.

\section{FIELD BUS INTERFACING}

Process Field Net abbreviated as PROFINET is an mechanical classic for bus interface data transmission \& transfer using professional Ethernet. It is developed for extracting data's through the I/O modules and controlling all the equipment in an industrial area. It has enhanced potential vitality in executing data under tight time constraints for a meagre of less than or equal to $1 \mathrm{~ms}$. The PROFINET initiates auto-negotiation with the CPU 410 AS Smart. The PROFINET requires Full Duplex mode of operation and also allows ETHERNET communication with it. The Auto negotiation forces the CPU to adapt to the settings of the partner device coupled with it. As PROFINET I/O demands 100 Mbps full-duplex operation, the ETHERNET communication cannot be used for long term option to address the I/O devices, because the ETHERNET operated at 10 Mbps half-duplex mode.

\section{OPERATION TECHNIQUE}

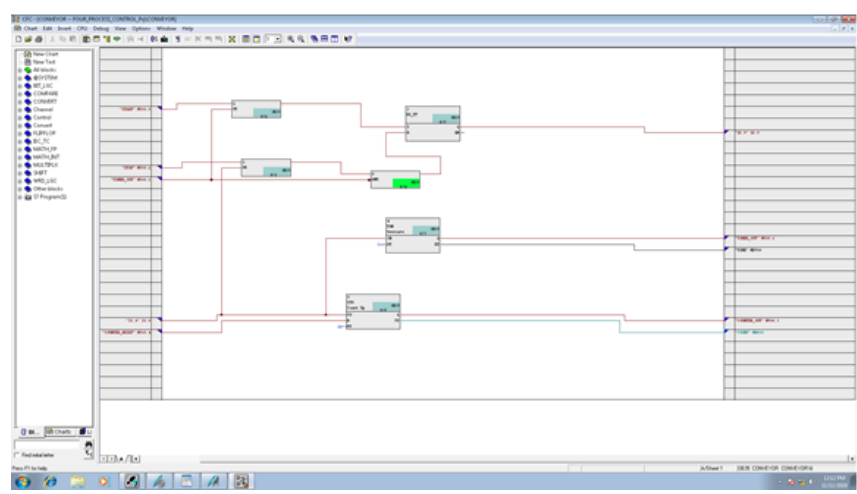

Fig. 1.Functinoal block diagram using SCADA

The materials in the conveyor belt are sorted using RFID and the photoelectric proximity sensor. First the logic of material sorting is programmed into the PCS 7 system through the PROFINET bus interface. The functional block diagram of the material sorting is drawn in the CFC chart of the SIEMATIC Manager software in the computer system. The CPU 410AS Smart and the computer system and inter linked through the PROFINET field BUS protocol.

The functional block diagram is stimulated in the SCADA screen linked in the plant view of the SIEMATIC manager. The SCADA screen is generated by inbuild library functions of the SIMATIC Manager software. The simulation when done properly is moved on to the next step of field automation. Through the field bus interface, the functional block program is loaded to the CPU 410 AS Smart. Then through the PROFINET protocol the ET200SP module is interfaced with the field devices such as RFID and photoelectric proximity sensor, conveyor belt and stepper motor. According to the program loaded to the DCS the RFID detects the material and sorts it based on the incoming materials in the conveyor belt. This type of material sorting enables error free performance and the latency time required for iterating the operating is hugely minimized to spontaneous response of the dcs and the RFID. In industrial and medical field this type of error free and spontaneous response improves the overall performance and the efficiency of the system thereby the systems reliance gets multiplied to manifold.

\section{RESULTS}

The conveyor belt material sorting is simulated using the SCADA screen in the SIMATIC Manager software and confirmed for its reduced response time and high efficiency.

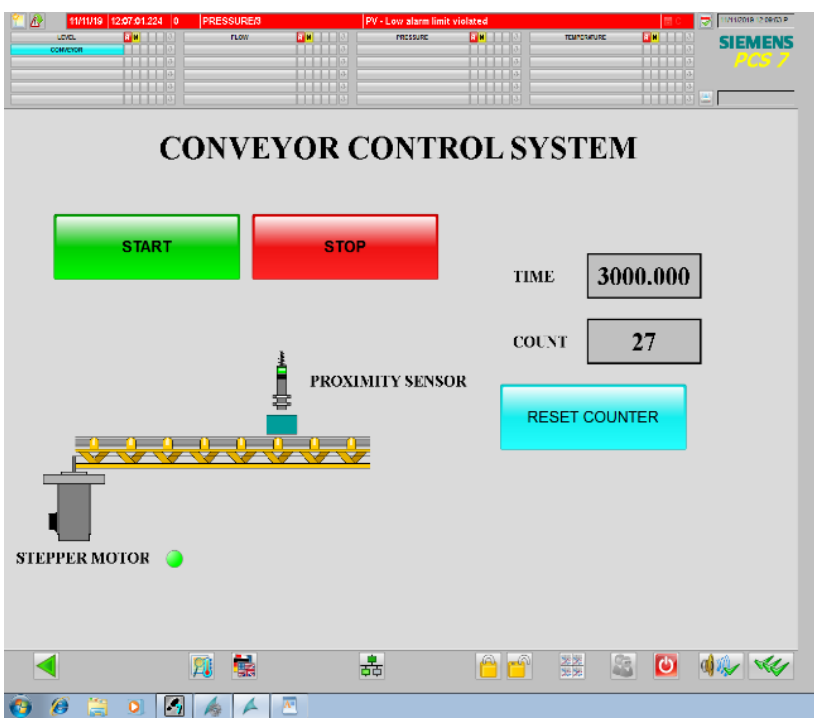

Fig. 2.Simulation of conveyor belt using SCADA 


\section{RFID Based Material Sorting System using Siemens DCS Controller}

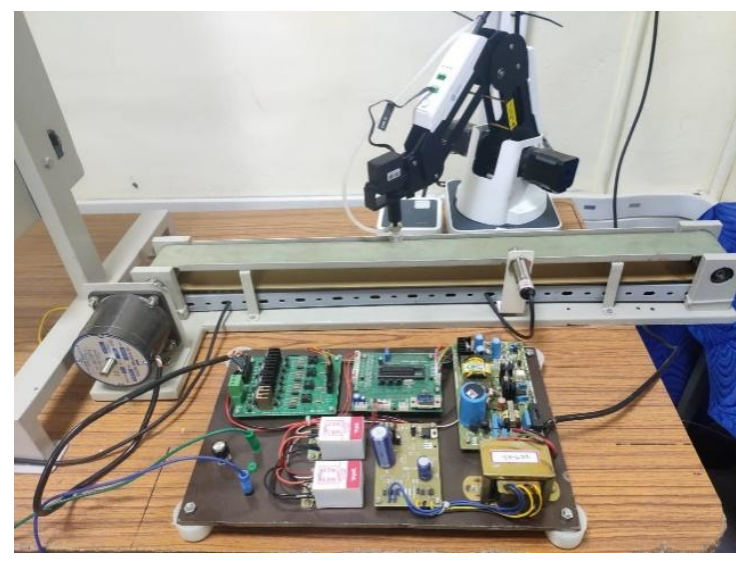

Fig. 3. Hardware setup

\section{CONCLUSION}

The field bus distributed control technique offers a wide range of enhanced automated solutions and improved efficiency in industrial sector. The inclusion of DCS in the controlling mechanism makes the system more reliable irrespective of the load relayed on the conveyor belt. Energy management can also be made possible using DCS and PCS7 components with some modification in the functional block diagram by using priority-based methods. Thus, the real time monitoring and controlling of on \& off operation and the material segregation of variant load is achieved using DCS in a long run producing improved latency and increased efficiency.

\section{REFERENCES}

1. James or Lin Qing Dapeng Tien, "Home automation using DCS technology", Department of Electrical Engineering, Ngee Ann Polytechnic, Singapore Copyright (C) IFAC Distributed Computer Control Systems. Seoul. Korea, 1997

2. West Szer-Ming Lee, Robert Harrison \& Andrew A. Wesl, "A Component Based Distributed Control System for Assembly automation”, MSI Research Institute, Loughborough University, United Kingdom, LEI 13 TU

3. S.P.S. Gill, N.K. Suriyadevara \& S.C. Mukhopadhyay "Smart Power Monitoring System using Wireless sensor networks", Massey University, Palmerston North, New Zealand, 978-1-4577-0167-2/12/\$26.00@ 2012 IEEE

4. Prakruthi Yadav, Vijay N. Chavda "Development of Power Monitoring and Analysis system based on Web server" "International Journal of Engineering Development and Research(www.ijedr.org) 1840" IJEDR| volume 2, issue 2| ISSN; 2321-939 IJEDR1402089 @ 2014.

5. Wisam Nader, "Real-time power monitoring, Home Automation and sustainability".

6. George Hassabis "Component-based engineering of Distributed control and automation software", "IEEE Jordan conference on Applied Electrical Engineering and Computing technologies (AEECT)" the IEC 61499 standard Department of Electrical and Computer Engineering, Aristotle University of Thessaloniki Thessaloniki, Greece 2015

7. Amir Firoozshahi "Innovative Intelligent DCS based methods for tank gauging control system in large oil terminal" IEEE International Symposium on Industrial electronics (ISIE 2009) Seoul Olympic Parktel Seoul Korea July 5-8 pp 1925-1931 July 2009.

8. J. Jeyapadmini K.R. Kashwan "Effective power utilization and Conservation in Smart Homes using IoT" 2015 International conference on Computation of Power Information and Communication 2015.

9. Mr. Pranay P. Gaikwad, Mrs. Jyotsna P. Gabhane, Mrs. Snehal S. Golait "A Survey based on smart home system using Internet-of-things" International Conference on Computation of Power Information and Communication, 2015.
10. M.Mastali A. A, Safavi H, A. Palizban "An easy to interpret fault detection approach to mutli variable statistical process monitoring" 16th Iranian conference on electric Engineering ,2008.

\section{AUTHORS PROFILE}

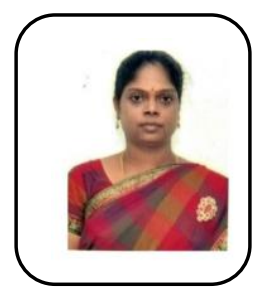

L. Chitra is an Associate Professor and Head in the department of EEE at Aarupadai Veedu Institute of Technology. She received her Bachelor Degree in Electronics and Instrumentation Engineering. She received her Master's Degree in Power Electronics and Drives in Anna University. She completed her $\mathrm{PhD}$ in Sathyabama Institute of Science and Technology in the field of Micro Electro Mechanical Systems for sensor design. Her research area includes MEMS, Renewable Energy, Process control, Sensors and Smart Sensors. She has published more than fifteen papers in International Conferences and Journals. She has a teaching experience of more than 18 years. She has received MNRE fund of Rs.1,50,000 for National Conference on Emerging Trends in New Renewable Energy Sources in the year 2014. She has also received a fund of Rs.1,00,000 from Unnat Bharat Abhiyan for Mobile Reverse osmosis based water purification system using Solar PV source for rural India in year 2019. She has also received a fund of Rs.50,000 from Unnat Bharat Abhiyan for DC Micro Grid based Solar PV generation to supply power to street lamp and common utilities in a village.

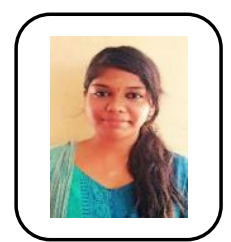

Sunkrutha S was born in 1998 in India. She did her schooling in Sri Sitaram Vidyalaya, Chennai. Currently she is pursuing her final year, bachelor of Engineering Degree in Mechatronics from Aarupadai Veedu Instituite of Technology which is part of Vinayaka Missions Research Foundation. Her current research interest includes Distributed control system, PLC, Renewable Energy Utilization, Sensors and Automation. She was conferred with the title of young women achiever from VMRF for her academic credentials. Her proposed research works include automated panel cleaner and DCS based Smart metering and controlling. She went to Thailand's Naresuan University as a part of student exchange program. She got the best spot presentation award in IEI conference held in Chennai.

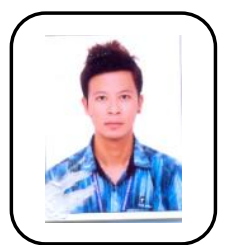

Prashant Pradhan, born in Chennai, He has completed his Bachelor's Degree at Aarupadai Veedu Institute of Technology, Vinayaka Mission's Research Foundation, Chennai. He has worked on many projects related to Renewable Energy, PLC, DCS, RFID controller etc.,. He is one of the notable student in his college, for his interest in the field of Automation. His current research interests include power electronics, renewable energy, power transmission, PLC, DCS, etc., 